\title{
Oxidative Damage and Antioxidant Defense in Ferroptosis
}

\author{
Feimei Kuang ${ }^{1}$, Jiao Liu', Daolin Tang ${ }^{1,2 *}$ and Rui Kang ${ }^{2 *}$ \\ ${ }^{1}$ The Third Affiliated Hospital, Guangzhou Medical University, Guangzhou, China, ${ }^{2}$ Department of Surgery, UT Southwestern \\ Medical Center, Dallas, TX, United States
}

OPEN ACCESS

Edited by:

Yinan Gong,

University of Pittsburgh, United States

Reviewed by:

Jennifer Martinez,

National Institute of Environmental Health Sciences (NIEHS),

United States

Giovanni Quarato,

St. Jude Children's Research

Hospital, United States

${ }^{*}$ Correspondence:

Daolin Tang

daolin.tang@utsouthwestern.edu

Rui Kang

rui.kang@utsouthwestern.edu

Specialty section:

This article was submitted to Cell Death and Survival,

a section of the journal

Frontiers in Cell and Developmental

Biology

Received: 23 July 2020

Accepted: 28 August 2020

Published: 17 September 2020

Citation:

Kuang F, Liu J, Tang D and

Kang R (2020) Oxidative Damage

and Antioxidant Defense

in Ferroptosis.

Front. Cell Dev. Biol. 8:586578.

doi: $10.3389 /$ fcell.2020.586578
Many new types of regulated cell death have been recently implicated in human health and disease. These regulated cell deaths have different morphological, genetic, biochemical, and functional hallmarks. Ferroptosis was originally described as a carcinogenic RAS-dependent non-apoptotic cell death, and is now defined as a type of regulated necrosis characterized by iron accumulation, lipid peroxidation, and the release of damage-associated molecular patterns (DAMPs). Multiple oxidative and antioxidant systems, acting together autophagy machinery, shape the process of lipid peroxidation during ferroptosis. In particular, the production of reactive oxygen species (ROS) that depends on the activity of nicotinamide adenine dinucleotide phosphate (NADPH) oxidases (NOXs) and the mitochondrial respiratory chain promotes lipid peroxidation by lipoxygenase (ALOX) or cytochrome $\mathrm{P} 450$ reductase (POR). In contrast, the glutathione $(\mathrm{GSH})$, coenzyme Q10 (CoQ10), and tetrahydrobiopterin $\left(\mathrm{BH}_{4}\right)$ system limits oxidative damage during ferroptosis. These antioxidant processes are further transcriptionally regulated by nuclear factor, erythroid 2-like 2 (NFE2L2/NRF2), whereas membrane repair during ferroptotic damage requires the activation of endosomal sorting complexes required for transport (ESCRT)-III. A further understanding of the process and function of ferroptosis may provide precise treatment strategies for disease.

\section{Keywords: ferroptosis, cell death, ROS, antioxidant, redox}

\section{INTRODUCTION}

The survival and death of cells are strictly controlled by various signals and molecules (Fulda et al., 2010). Physiological cell death is essential for normal function and tissue development. But pathological cell death may have side effects that cause inflammation and threaten our health. The first classification of cell death was based on morphological criteria proposed by pathologists in the 1970s. The pathologists suggested that cell death has three main forms, namely apoptosis (type I), autophagy (type II), and necrosis (type III) (Schweichel and Merker, 1973). The typical morphological changes of apoptosis are nuclear chromatin concentration and the formation of apoptotic bodies in the cytoplasm (Elmore, 2007). This is different from cell swelling and membrane rupture in necrosis (Golstein and Kroemer, 2007) and the formation of cytoplasmic double membrane vesicles in autophagy (a lysosome-dependent degradation process) (Xie et al., 2015). The latest cell death classification was formulated and is recommended by the Cell Death Nomenclature Committee. Generally, cell death is divided into accidental cell death and regulated cell death (Galluzzi et al., 2018). Accidental cell death is a passive process, whereas regulated 
cell death is an active process that plays an important role in the pathogenesis of the disease (Galluzzi et al., 2018). In the past 20 years, many new types of regulated cell death (e.g., necroptosis, pyroptosis, ferroptosis, entotic cell death, netotic cell death, parthanatos, lysosome-dependent cell death, autophagy-dependent cell death, alkaliptosis, and oxeiptosis) have been identified in various models (Tang et al., 2019). Although they may share several common signals (e.g., redox signals), different forms of regulated cell death require special molecular machinery to trigger cell death (Tang et al., 2019). In this review, we summarize the major ways in which oxidative stress and antioxidant defense regulate ferroptosis, which is a form of iron-dependent cell death driven by lipid peroxidation (Figure 1).

\section{THE BASIC PROPERTIES OF FERROPTOSIS}

The concept of ferroptosis is derived from precision medicine for tumors that targeted RAS mutation signals (Dolma et al., 2003; Yang and Stockwell, 2008). RAS is a proto-oncogene and is frequently mutated in human cancer, leading to tumorgenesis and therapy resistance. Drug screening identified that the small molecular compounds erastin and RSL3 can selectively kill RASmutant cancer cells, but not RAS wild-type cells (Dolma et al., 2003; Yang and Stockwell, 2008). Later, it was proved that the anticancer activity of erastin and RSL3 depends on the induction of a new type of iron-dependent cell death, termed ferroptosis (Dixon et al., 2012). Although initial research showed that ferroptosis may be different from the classical cell death (e.g., apoptosis, necrosis, and autophagy-dependent cell death) (Dixon et al., 2012), recent studies demonstrate that there is a close relationship between ferroptosis, necrosis, and autophagy (Chen M.S. et al., 2017; Muller et al., 2017; Li et al., 2020; Lin et al., 2020; Liu et al., 2020c; Xie et al., 2020a). Ferroptotic cells usually exhibit cell membrane rupture and the release of intracellular contents, especially damage-associated molecular patterns (DAMPs) (Wen et al., 2019; Dai et al., 2020b), and are therefore classified as a type of regulated necrosis (Conrad et al., 2016). Increased autophagy, especially several types of selective autophagy [e.g., ferritinophagy (Hou et al., 2016), lipophagy (Bai et al., 2019), clockophagy (Yang M. et al., 2019), and chaperonemediated autophagy ( $\mathrm{Wu}$ et al., 2019)], promotes ferroptosis, indicating that ferroptosis is related to an abnormal intracellular degradation pathway.

Although the direct effectors of ferroptosis are unclear, iron accumulation and lipid peroxidation seem to play a central role in regulating the process of ferroptosis (Dixon et al., 2012; Yang et al., 2016; Shintoku et al., 2017; Wenzel et al., 2017; Li et al., 2020). Iron is an essential nutrient for cell proliferation, but iron overload can cause iron toxicity and lead to cell damage, even death. The balance of iron in cells and in the body is controlled by an integrated system. Pathways for abnormal iron metabolism, such as increasing the iron absorption and reducing iron storage or iron output, may cause ferroptosis through at least two ways. One is iron-mediated reactive oxygen species (ROS) production through the Fenton reaction (Dixon et al., 2012). The other is involved in the activation of iron-containing enzymes, such as lipoxygenase (ALOX) (Yang et al., 2016; Shintoku et al., 2017; Wenzel et al., 2017; Li et al., 2020). Finally, the accumulation of iron causes lipid peroxidation, which is the process of oxidative degradation in lipids [especially polyunsaturated fatty acids (PUFAs)] (Yuan et al., 2016; Doll et al., 2017; Kagan et al., 2017), leading to subsequent membrane damage and rupture. In contrast, an increased membrane repair ability through the activation of charged multivesicular body protein 5 (CHMP5) and charged multivesicular body protein 6 (CHMP6), which then mediate the endosomal sorting complexes required for transport (ESCRT)-III pathway, limits ferroptosis (Dai et al., 2020c). Notably, multiple oxidative stress and antioxidant defense pathways are involved in shaping ferroptotic responses (discussed later). This process is further regulated by epigenetic, transcriptional, posttranscriptional, and posttranslational mechanisms (Dai et al., 2020a; Wu et al., 2020). In particular, the activation of nuclear factor, erythroid 2like 2 (NFE2L2/NRF2) plays a major transcriptional regulatory role in the suppression of ferroptosis through the induction of expression of antioxidants or iron metabolism genes (Sun et al., 2016a,b). Functionally, impaired ferroptosis (due to its excessive activation or cellular defects) is increasingly recognized as the cause of human diseases, especially neurodegenerative diseases, cancer, and infectious diseases as well as tissue damage (Xie et al., 2016; Stockwell et al., 2017).

\section{OXIDATIVE DAMAGE IN FERROPTOSIS}

Oxidative damage results from an imbalance between the generation of free radicals and the body's ability to neutralize or eliminate their harmful effects through antioxidants. ROSmediated lipid peroxidation is the key step that drives ferroptosis. Below, we describe the main cellular sources of ROS and the main regulators of lipid peroxidation during ferroptosis (Figure 1).

\section{Mitochondria-Mediated ROS Production}

Mitochondria play a key role in regulating cell energy and cell death signal transduction. In addition to producing adenosine triphosphate, mitochondria are also the main source of ROS production (Zorov et al., 2014). The production of mitochondrial ROS mainly occurs during the oxidative phosphorylation in the electron transport chain located on the inner membrane of the mitochondria. Electrons leak from complex I and complex III on the electron transport chain, resulting in a partial reduction of oxygen to form superoxide anion $\left(\mathrm{O}_{2}{ }^{-}\right)$. Subsequently, $\mathrm{O}_{2}{ }^{--}$ is rapidly disproportionated into hydrogen peroxide $\left(\mathrm{H}_{2} \mathrm{O}_{2}\right)$ by the superoxide dismutase 2 (SOD2) in the mitochondrial matrix and superoxide dismutase 1 (SOD1) in the intermembrane space. Overall, $\mathrm{O}_{2}{ }^{-}$and $\mathrm{H}_{2} \mathrm{O}_{2}$ produced during this process are called mitochondrial ROS, which is related to the loss of mitochondrial membrane potential ( $\Delta \Psi \mathrm{m})$ (Zorov et al., 2014). The reduction of $\Delta \Psi \mathrm{m}$ is a common sign of apoptosis and ferroptosis, but their regulatory mechanisms are different. In 


\section{Proferroptotic pathway $\quad$ Antiferroptotic pathway}

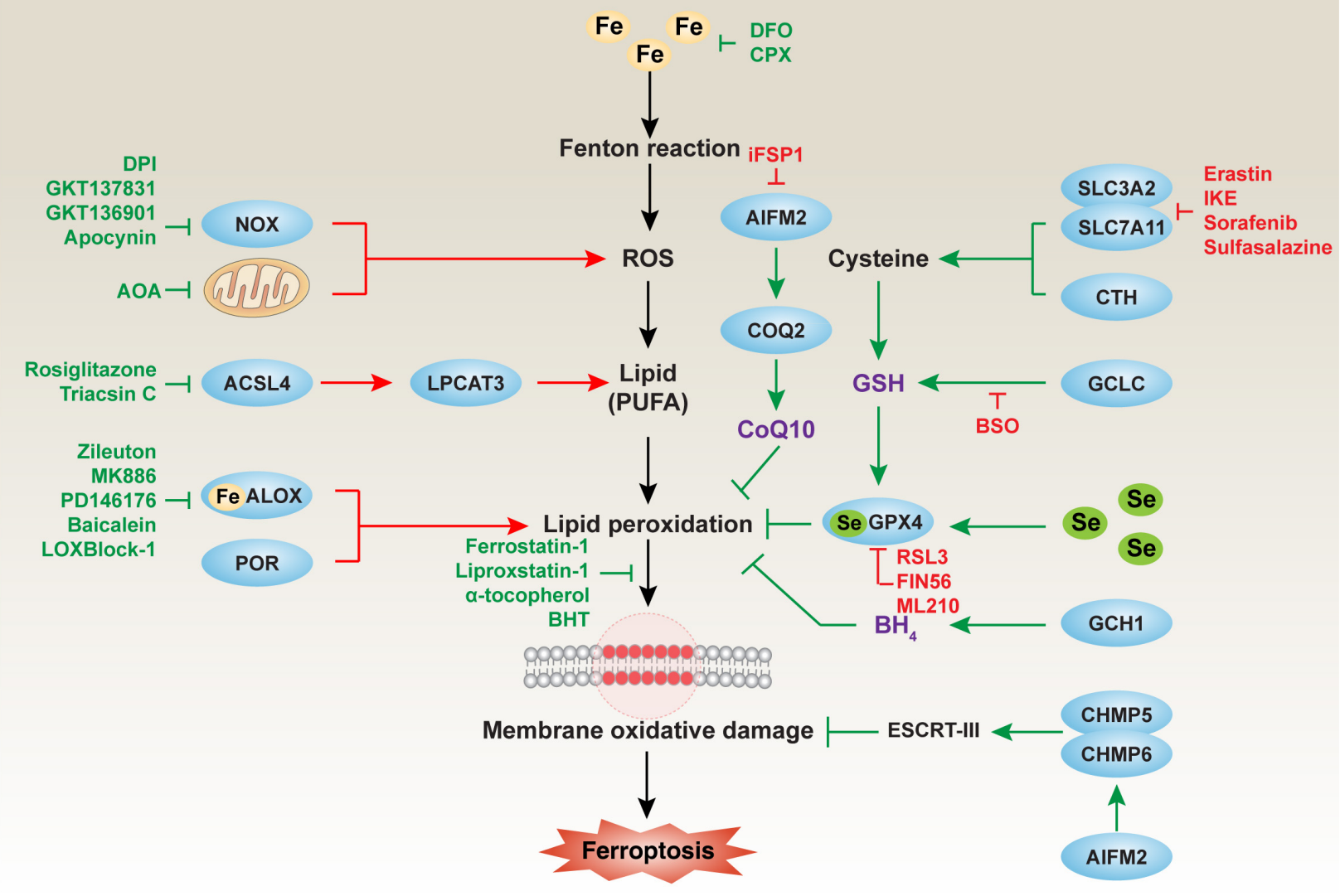

FIGURE 1 | The major mechanism of oxidative damage and antioxidant defense in ferroptosis. Ferroptosis is an iron-dependent oxidative cell death caused by ROS from the Fenton reaction and subsequent lipid peroxidation. Multiple oxidative and antioxidant systems control the process of membrane oxidative damage during ferroptosis. In particular, NOX-dependent and mitochondrial respiratory chain-dependent ROS production promotes lipid peroxidation by ALOX or POR. The production of lipids, especially PUFA, requires the activation of the ACSL4-LPCAT3 pathway. In contrast, the GSH, CoQ10, and BH 4 systems limit oxidative damage during ferroptosis. Membrane repair during ferroptotic damage requires the activation of the ESCRT-III pathway. ACSL4, acyl-CoA synthetase long-chain family member 4; AIFM2, apoptosis-inducing factor mitochondria-associated 2; ALOX, lipoxygenase; $\mathrm{BH}_{4}$, tetrahydrobiopterin; CHMP5, charged multivesicular body protein 5; CHMP6, charged multivesicular body protein 6; COQ2, coenzyme Q2, polyprenyltransferase; CoQ10, coenzyme Q10; CTH, cystathionine gamma-lyase; ESCRT-III, endosomal sorting complexes required for transport-III; GCH1, GTP cyclohydrolase 1; GCLC, glutamate-cysteine ligase catalytic subunit; GPX4, glutathione peroxidase 4; GSH, glutathione; LPCAT3, lysophosphatidylcholine acyltransferase 3; NOX, nicotinamide adenine dinucleotide phosphate (NADPH) oxidase; POR, cytochrome P450 oxidoreductase; ROS, reactive oxygen species; Se, selenium; SLC3A2, solute carrier family 3 member 2; SLC7A11, solute carrier family 7 member 11.

mitochondria, cytochrome C, somatic (CYCS) plays an essential role in generating $\Delta \Psi \mathrm{m}$. The translocation of CYCS from the mitochondria to the cytoplasm is an important event that initiates the loss of $\Delta \Psi \mathrm{m}$ in the process of apoptosis induced by mitochondrial ROS (Yang et al., 1997). However, changes in CYCS position and the subsequent activation of apoptosis effector caspases are not observed during ferroptosis (Dixon et al., 2012), indicating that different mechanisms control $\Delta \Psi \mathrm{m}$ during ferroptosis.

Some important mitochondrial apoptosis regulators, such as those in the voltage-dependent anion channel (VDAC) family and BCL2 family, are also involved in the regulation of ferroptosis in a context-dependent manner. VDAC, also known as mitochondrial porin, is the most abundant protein in the outer mitochondrial membrane. In the VDAC family, VDAC2 and VDAC3 are considered to be directly targeted by erastin to induce ferroptosis (Figure 2; Yagoda et al., 2007). As a negative feedback mechanism, the proteasome degradation of VDAC2 and VDAC3 that depends on NEDD4 E3 ubiquitin protein ligase can block erastin-induced ferroptosis in melanoma cells (Yang et al., 2020b). NEDD4-like E3 ubiquitin protein ligase (NEDD4L) inhibits ferroptosis by degrading lactotransferrin protein (Wang et al., 2020). VDAC2 is also a direct target of lipid-derived electrophile-induced carbonylation proteins during ferroptosis caused by RSL3 (Chen et al., 2018). Therefore, posttranslational modulation of VDAC2 or VDAC3 may weaken or exacerbate ferroptosis sensitivity. In addition to VDAC, the BCL2 family is also an important regulator of mitochondrial outer membrane permeability, which consists of many members that promote or inhibit apoptosis (Youle and Strasser, 2008). Pro-apoptotic BCL2 


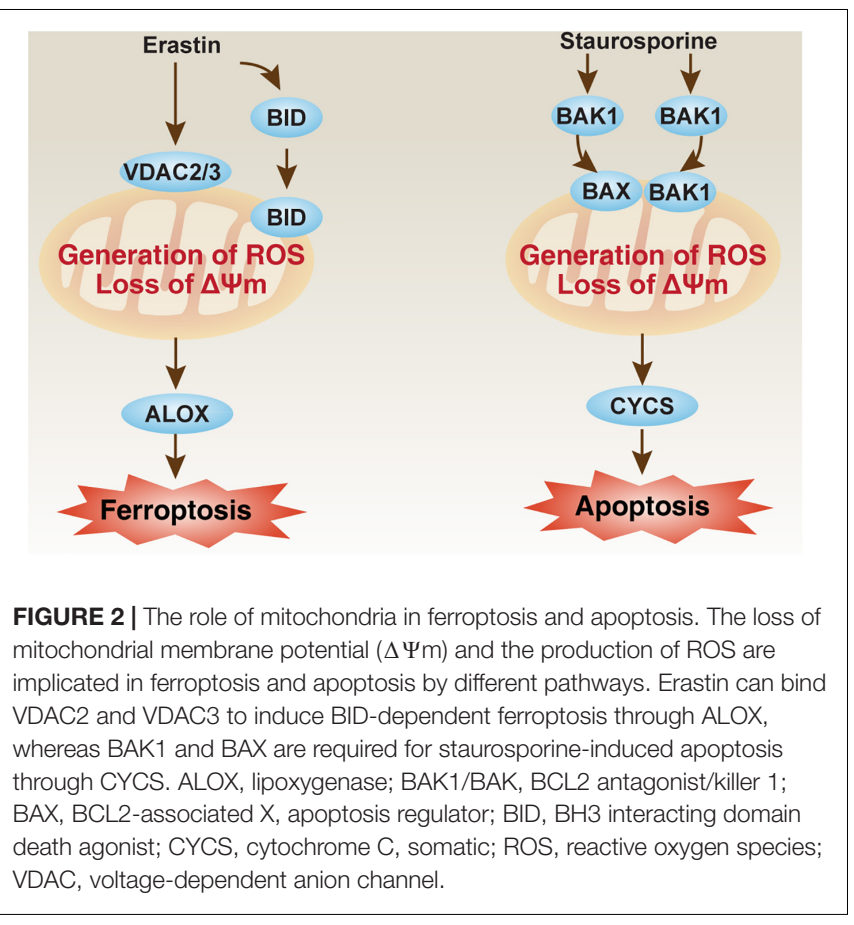

family members, such as BCL2 antagonist/killer 1 (BAK1/BAK) and BCL2-associated X, apoptosis regulator (BAX), are required for staurosporine-induced apoptosis (Wei et al., 2001), but not required for erastin-induced ferroptosis in fibroblasts (Dixon et al., 2012; Figure 2). However, BH3 interacting domain death agonist (BID), a pro-apoptotic BCL2 family member, mediates mitochondrial ROS-induced ferroptosis in neuronal cells (Neitemeier et al., 2017; Jelinek et al., 2018). These findings increase the likelihood that apoptotic or ferroptotic death requires different types of changes in mitochondrial membrane, which are further regulated by different members of VDAC and BCL2 families.

In addition, mitochondrial energy sensors or mitochondrial quality control systems plays a dual role in ferroptosis. AMP-activated protein kinase (AMPK) is a key sensor of cellular energy and regulates ferroptosis through its phosphorylated substrate. AMPK-mediated BECN1 (a key autophagy regulator) phosphorylation promotes ferroptosis through the inhibition of system $\mathrm{xc}^{-}$activity (Song et al., 2018). In contrast, AMPK-mediated acetyl-CoA carboxylase alpha (ACACA) phosphorylation blocks ferroptosis through the inhibition of fatty acid biosynthesis (Lee et al., 2020). Additional signals, currently unknown, are needed to explain the substrate selectivity of AMPK-related ferroptosis regulation. Although many types of selective autophagy promote ferroptosis, mitophagy (an important mitochondrial quality control system) may play a context-dependent role in ferroptosis. On the one hand, mitophagy can maintain a healthy number of mitochondria to promote survival against ferroptosis (Gao et al., 2019). On the other hand, excessive mitophagy may cause metabolic stress and subsequent production of mitochondrial ROS, leading to ferroptosis (Basit et al., 2017). The interaction of mitochondria and other organelles in ferroptosis remains to be explored.

\section{NOX-Mediated ROS Production}

For a long time, the production of $\mathrm{O}_{2}{ }^{--}$by transmembrane nicotinamide adenine dinucleotide phosphate (NADPH) oxidase (NOX) has been regarded as an important function in professional phagocytes, such as macrophages and dendritic cells (Bedard and Krause, 2007). In addition to phagocytes, other cells also express NOX to produce $\mathrm{O}_{2}{ }^{-}$or $\mathrm{H}_{2} \mathrm{O}_{2}$ by transporting electrons across the membrane. The human genome encodes seven members of the NOX family, including five NOX proteins [NOX1, cytochrome B-245 beta chain (CYBB/NOX2), NOX3, NOX4, and NOX5] and two dual oxidases (DUOX1 and DUOX2). NOX-derived ROS plays a broad role in various physiological and pathological conditions (e.g., development, infection, immunity, and cell death) (Bedard and Krause, 2007). As an important regulator of lipid raft-derived redox signaling platforms, NOX participates in the induction of apoptosis (Jin et al., 2011). Similarly, NOX1-, CYBB-, and NOX4-mediated ROS production is also involved in the initiation of ferroptotic cancer cell death by inducing lipid peroxidation (Xie et al., 2017; Chen et al., 2019; Yang W.H. et al., 2019; Yang et al., 2020a), indicating a wide role for NOXs in cell death. In cancer cells, the activity of NOXs in ferroptosis is further affected by oncogenes and tumor suppressors. For example, the loss of tumor suppressor TP53 inhibits the accumulation of dipeptidyl-peptidase-4 (DPP4/CD26) in the nucleus, thereby increasing plasma-membrane associated DPP4-dependent lipid peroxidation and subsequent ferroptosis via the formation of the DPP4-NOX1 complex (Xie et al., 2017). During the activation of oncogenic RAS, NOX1 mediates the production of ROS (Adachi et al., 2008), which may promote ferroptosis through the activation of the extracellular signal-regulated kinase (ERK) pathway (Yagoda et al., 2007). More research is needed to clearly define how different NOX members, coupled with impaired genetic signals in tumors, cause ferroptosis.

\section{ALOX-Mediated Lipid Peroxidation}

Reactive oxygen species-mediated lipid peroxidation is mainly accomplished by ALOX, which is a dioxygenase that contains non-heme iron. ALOX includes six members (ALOXE3, ALOX5, ALOX12, ALOX12B, ALOX15, and ALOX15B) and catalyzes the stereotactic insertion of oxygen into PUFAs, especially arachidonic acid (AA) and adrenic acid (AdA), in a tissue- or cell-dependent manner. For example, ALOX5, ALOXE3, ALOX15, or ALOX15B mediates ferroptosis caused by erastin or RSL3 in BJeLR, HT1080, or PANC1 cells (Yang et al., 2016; Shintoku et al., 2017; Wenzel et al., 2017; Li et al., 2020). ALOX15 or ALOX12 mediates TP53-induced ferroptosis in cancer cells following different stimuli (Ou et al., 2016; Chu et al., 2019). Phosphatidylethanolaminebinding protein 1 (PEBP1) can be used as an adaptor protein for ALOX15 and enhances the activity of ALOX15 in the induction of ferroptosis in vitro (Wenzel et al., 2017). However, ALOX12/15 may not be important for ferroptotic damage in mice caused by glutathione peroxidase 4 (GPX4) 
depletion in kidney (Friedmann Angeli et al., 2014) or T cells (Matsushita et al., 2015). Therefore, when evaluating the sensitivity of ferroptosis, it is necessary to first detect the basic expression levels of different ALOX members.

Lipoxygenase-mediated lipid peroxidation is firstly initiated by the generation of AA/AdA derivatives mediated by ACSL4 and LPCAT3 (Yuan et al., 2016; Doll et al., 2017; Kagan et al., 2017). ACSL4 catalyzes the combination of free AA/AdA and $\mathrm{CoA}$ to form $\mathrm{AA} / \mathrm{AdA}-\mathrm{CoA}$ derivatives and promotes their esterification to phospholipids, while LPCAT3 then catalyzes the biosynthesis of AA/AdA-CoA and membrane phosphatidylethanolamine (PE) to form AA/AdA-PE. ALOX then mediates the peroxidation of AA/AdA-PE to generate AA/AdA-PE-OOH (e.g., 5-HETE, 11-HETE, and 15-HETE, but not 12-HETE), which leads to membrane injury during ferroptosis. Therefore, the genetic or pharmacological inhibition of the ACSL4-LPCAT3-ALOX pathway inhibits ferroptosis in vitro and in vivo. Although ACSL4-independent ferroptosis may exist, increased ACSL4 expression is a biomarker of ferroptosis (Yuan et al., 2016).

\section{POR-Mediated Lipid Peroxidation}

Cytochrome P450 reductase (POR) plays a major role in the metabolism of drugs and steroids. POR supplies electrons to microsomal cytochrome P450 from NADPH (Riddick et al., 2013). Consequently, the destruction of POR affects the activity of all microsomal P450 enzymes. In addition to ALOX-mediated lipid peroxidation, POR-mediated lipid peroxidation plays an alternative role in mediating ferroptosis. In particular, POR binds its cofactors, such as flavin mononucleotide (FMN) and flavin adenine dinucleotide (FAD). This complex mediated electron supplementation to cytochrome P450 from NADPH is required for erastin-, FIN56-, ML210-, or RSL3-induced lipid peroxidation and subsequent ferroptosis in melanoma and other cancer cells (Zou et al., 2020). Although the exact mechanism of POR-mediated lipid peroxidation is still unknown, POR may accelerate the cycling between ferrous and ferric iron in the heme component of cytochrome P450 (Zou et al., 2020). Given that the conditional knockout of POR in the liver leads to a decrease in the metabolism and lipid accumulation in mice (Henderson et al., 2003), the pathological role of POR-mediated ferroptosis in tissue damage and metabolism disease is worthy of further study.

\section{ANTIOXIDANT DEFENSE IN FERROPTOSIS}

Cellular protection against oxidative damage in ferroptosis is organized at multiple levels. The synthesis of antioxidants, such as glutathione (GSH), coenzyme Q10 (CoQ10) and tetrahydrobiopterin $\left(\mathrm{BH}_{4}\right)$, is the main defense strategy in the process of ferroptosis, which is related to multiple enzymes or proteins (Figure 1). In addition to the antioxidant systems discussed below, some antioxidant proteins, such as peroxiredoxins (PRDXs) (Lu et al., 2019; Qi et al., 2019; Lovatt et al., 2020) and thioredoxin (Llabani et al., 2019), can also block ferroptotic cell death. Therefore, an integrated antioxidant defense network exists in different cells.

\section{GSH System}

Glutathione is an active tripeptide, formed by the condensation of glutamic acid, cysteine, and glycine. As an important antioxidant, glutathione is used to treat liver diseases, tumors, poisoning, cataracts, and aging diseases. The pharmacological inhibition of GSH synthesis and utilization is a classic method of inducing ferroptosis (Dixon et al., 2012; Yang et al., 2014). There are two main sources of cysteine production for GSH synthesis, namely the system $\mathrm{xc}^{-}$pathway and the transsulfuration pathway (McBean, 2012; Lewerenz et al., 2013). System $\mathrm{xc}^{-}$is a transmembrane sodium-independent and chloride-independent transporter of cystine and glutamic acid, which contains two key components [solute carrier family 7 member 11 (SLC7A11/xCT) and solute carrier family 3 member 2 (SLC3A2/CD98)] (Lewerenz et al., 2013). After being transported into the cell by system $\mathrm{xc}^{-}$, cystine is oxidized to cysteine, which is then used for glutamate-cysteine ligase catalytic subunit (GCLC/GCL)-mediated GSH synthesis. The inhibition of system $\mathrm{xc}^{-}$(using erastin, sorafenib, and sulfasalazine) or GCL (using buthionine sulfoximine) triggers ferroptosis in various cells (Conrad and Pratt, 2019). The expression or activity of system $\mathrm{xc}^{-}$is affected by epigenetics, transcription, and posttranscriptional and posttranslational regulators, such as TP53 (Jiang et al., 2015), NFE2L2 (Chen D. et al., 2017), BRCA1-associated protein 1 (BAP1) (Zhang Y. et al., 2018), mucin 1, cell surface-associated (MUC1) (Hasegawa et al., 2016), or BECN1 (Song et al., 2018), leading to complex feedback mechanisms to control GSH levels in ferroptosis. The transsulfuration pathway is a metabolic pathway that involves the interconversion of cysteine and homocysteine through intermediate cystathionine (McBean, 2012). Cystathionine gamma-lyase (CTH/CGL)-mediated decomposition of cystathionine is required for cysteine production. This process is inhibited by cysteinyl tRNA synthetase 1 (CARS1/CARS), an enzyme that charges tRNA ${ }^{\text {Cys }}$ with cysteine in the cytoplasm. In contrast, knocking down CARS1 increases resistance to ferroptosis by activating the transsulfuration pathway (Hayano et al., 2016).

The main anti-ferroptotic activity of GSH is related to GPX4, which reduces phospholipid hydroperoxide production (AA/AdA-PE-OOH) to AA/AdA-PE-OH (Yang et al., 2014). GPX4 is a selenium-containing protein whose activity is regulated by GSH. As an essential trace element, the function of selenium depends on a unique functional group, namely the selenol ($\mathrm{SeH}$ ) group. In the catalytic cycle of GPX4, active selenol ($\mathrm{SeH})$ is oxidized by peroxide to selenic acid $(-\mathrm{SeOH})$, and then reduced by GSH to intermediate selenide disulfide (-Se-SG) (Ingold et al., 2018). GPX4 is further activated by the second GSH, releasing glutathione disulfide (GS-SG) (Ingold et al., 2018). GPX4 inhibitors (e.g., RSL3, ML162, ML210, FIN56, and FINO2) are also known as classic ferroptosis activators, although their activities and effects are still different (Conrad and Pratt, 2019). In addition to ferroptosis, GPX4 also mediates antioxidant defense in apoptosis (Ran et al., 2003), necroptosis (Canli et al., 2016), and 
pyroptosis (Kang et al., 2018), suggesting a context-dependent role of GPX4 in cell death.

\section{CoQ10 System}

Coenzyme Q10 is a vitamin-like endogenously produced isoprenyl benzoquinone compound that occurs naturally in the human body and is highest in the heart, liver, kidney, and pancreas (Hernandez-Camacho et al., 2018). CoQ10 exists in oxidized form (ubiquinone) and reduced form (ubiquinol). The effective function of mitochondria depends on various cofactors, such as L-carnitine, $\alpha$-lipoic acid, and CoQ10 (Pagano et al., 2014). CoQ10 is particularly interesting because it not only supports the mitochondrial respiratory chain, but also acts as a powerful antioxidant by neutralizing free radicals in various membrane structures (Teran et al., 2018). CoQ10 not only inhibits apoptosis (Chen et al., 2011), but also ferroptosis (Shimada et al., 2016). For example, the application of farnesyl pyrophosphate (an upstream product of CoQ10 synthesis) or idebenone (a hydrophilic analog of CoQ10) prevents ferroptosis caused by FIN56 (Shimada et al., 2016). In contrast, inhibiting the production of CoQ10 may accelerate ferroptotic cell death.
In particular, apoptosis-inducing factor mitochondrial-related 2 (AIFM2/FSP1/AMID), a traditional regulator of apoptosis in the mitochondria (Wu et al., 2002), can mediate the production of CoQ10 to inhibit ferroptosis in a GSH-independent manner (Bersuker et al., 2019; Doll et al., 2019). This process requires the $\mathrm{N}$-myristoylation of AIFM2, which results in the translocation of AIFM2 to the cell membrane (Bersuker et al., 2019; Doll et al., 2019). The depletion of CoQ10 biosynthesis enzyme [e.g., coenzyme Q2, polyprenyltransferase (COQ2)] may reverse the anti-ferroptotic activity of AIFM2 (Bersuker et al., 2019; Doll et al., 2019). Of note, the increased accumulation of AIFM2 in the cell membrane may also inhibit ferroptosis by activating CHMP5- and CHMP6-mediated ESCRT-III membrane repair mechanisms, which are independent of CoQ10 (Dai et al., 2020d). Statin drugs inhibit 3-hydroxy-3-methylglutaryl-coenzyme A reductase (HMG-CoA), a rate-limiting step that converts HMG-CoA to mevalonate in the production of cholesterol. Interestingly, statins can cause a decrease in CoQ10, thereby increasing the sensitivity of ferroptosis (Shimada et al., 2016). Further understanding of the antioxidant capacity of CoQ10 may provide benefits for reducing ferroptosis-related damage.
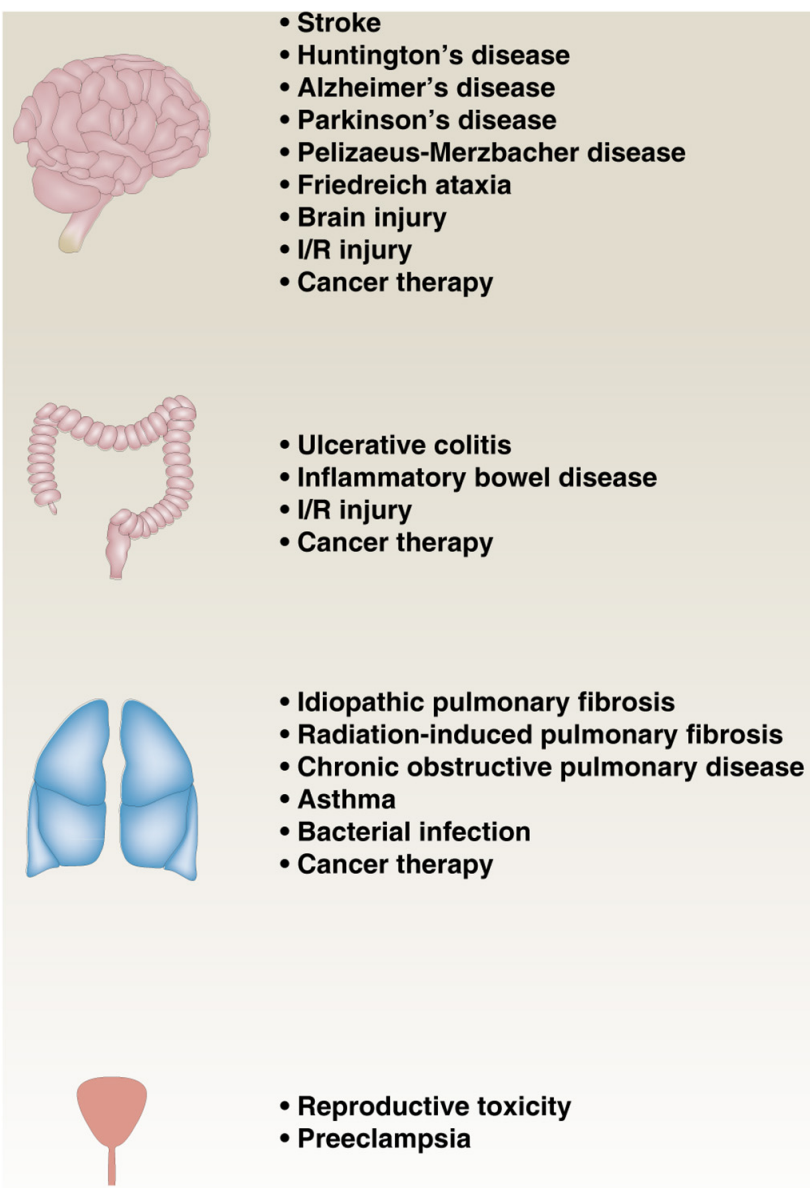

- Reproductive toxicity - Preeclampsia
- Drug injury

- I/R injury

- Parasite infection

- Hepatitis

- Nonalcoholic steatohepat

- Autoimmune hepatitis

- Liver fibrosis

- Cancer therapy

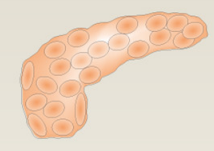

- Pancreatitis

- Cancer therapy

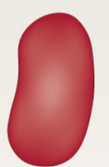

- Drug injury

- I/R injury

- Kidney failure

- Kidney cysts

- Kidney infection

- Diabetic kidney disease

- Cancer therapy

\section{- Drug injury \\ - I/R injury \\ - Heart failure \\ - Myocarditis}

FIGURE 3 | The role of ferroptosis in diseases. Impaired or excessive ferroptotic pathway is involved in various diseases, such as neurodegenerative diseases, infectious diseases, cancers, and ischemia-reperfusion (I/R) injury diseases. 


\section{$\mathrm{BH}_{\mathbf{4}}$ System}

Tetrahydrobiopterin is a natural nutrient that can be used as a cofactor for various enzymes, such as tryptophan hydroxylase, phenylalanine hydroxylase, tyrosine hydroxylase, nitric oxide (NO) synthase, and glyceryl ether mono-oxygenase (Werner et al., 2011). Functionally, $\mathrm{BH}_{4}$ is involved in the biosynthesis of some neurotransmitters, such as 5-hydroxytryptamine, dopamine, noradrenaline, adrenaline, and melatonin (Werner et al., 2011). Exogenous dopamine or melatonin has been shown to suppress erastin- or hemin-induced ferroptosis in various cells (NaveenKumar et al., 2019; Wang et al., 2016), but it remains unclear whether $\mathrm{BH}_{4}$-mediated endogenous dopamine or melatonin production regulates ferroptosis. In addition, $\mathrm{BH}_{4}$ plays a redox role in the catalysis of $\mathrm{L}$-arginine, $\mathrm{O}_{2}$, and $\mathrm{NADPH}$ to form NO. The oxidation of $\mathrm{BH}_{4}$ to $\mathrm{BH}_{2}$ causes an uncoupling of NOS, thereby forming $\mathrm{O}_{2}^{-}$instead of NO. $\mathrm{O}_{2} \cdot-$ reacts rapidly with $\mathrm{NO}$ to form peroxynitrite, and nitrite can further uncouple NOS (Werner et al., 2011). The activation of the NO pathway is implicated in ferroptosis-related tissue injury (Deng et al., 2020). In particular, L-arginine induces acute pancreatitis in mice through the activation of ferroptosis-induced sterile inflammation, which is further regulated by circadian rhythms (Liu et al., 2020d). The synthesis and recycling of $\mathrm{BH}_{4}$ is a dynamic process, and GTP cyclohydrolase-1 (GCH1) is the rate-limiting enzyme for the biosynthesis of $\mathrm{BH}_{4}$. $\mathrm{GCH} 1-$ mediated $\mathrm{BH}_{4}$ production prevents ferroptosis by inhibiting lipid peroxidation (Kraft et al., 2020), indicating that $\mathrm{BH}_{4}$ has antioxidant activity during cell death. Overall, these findings demonstrate the prosurvival role of $\mathrm{BH}_{4}$ in protecting against ferroptotic damage.

\section{FERROPTOSIS IN DISEASE}

More and more reports showing that impaired or excessive ferroptotic pathway in various diseases, such as neurodegenerative diseases, infectious diseases, cancers, and ischemia-reperfusion (I/R) injury diseases (Figure 3). In cancer pathology, ferroptosis not only inhibits tumor growth (Daher et al., 2019), but also promotes tumor formation (Dai et al., 2020b), depending on the type, stage, and microenvironment of the tumor. Iron-induced ferroptotic damage is implicated in Huntington's disease (HD), Alzheimer's disease (AD), and Parkinson's disease (PD) (Skouta et al., 2014; Do Van et al., 2016; Zhang Y.H. et al., 2018; Hirata et al., 2019), although oxytosis has long been considered to be the main mode leading to neuronal cell damage caused by glutamate toxicity (Tan et al., 2001). Inflammation mediated by ferroptotic cell death can promote pancreatitis (Liu et al., 2020d), liver fibrosis (Tsurusaki et al., 2019; Zeng et al., 2020), chronic obstructive

\section{REFERENCES}

Adachi, Y., Shibai, Y., Mitsushita, J., Shang, W. H., Hirose, K., and Kamata, T. (2008). Oncogenic Ras upregulates NADPH oxidase 1 gene expression through MEK-ERK-dependent phosphorylation of GATA-6. Oncogene 27, 4921-4932. doi: $10.1038 /$ onc. 2008.133 pulmonary disease (COPD) (Park et al., 2019; Wang and Tang, 2019; Yoshida et al., 2019), inflammatory bowel disease (Mayr et al., 2020), and preeclampsia (Zhang et al., 2020). In addition, inhibiting ferroptosis can prevent I/R damage to various tissues, especially liver, kidney, brain, and heart (Linkermann et al., 2014; Skouta et al., 2014; Martin-Sanchez et al., 2017; Muller et al., 2017; Fang et al., 2019). Therefore, the development of pharmacological agents that regulate ferroptosis under these pathological conditions is crucial.

\section{CONCLUSION AND PERSPECTIVES}

Ferroptosis was discovered not long ago, and there have been more and more studies related to it in recent years. This is because the core signals of ferroptosis (iron accumulation and lipid peroxidation) are often observed abnormally in various diseases and pathological conditions. Like other types of regulated cell death, ferroptosis may be caused by an imbalance between oxidation and antioxidant systems (Chen et al., 2020). In particular, NOX-dependent and mitochondrial respiratory chain-dependent ROS formation facilitates lipid peroxidation, whereas the $\mathrm{GSH}, \mathrm{CoQ} 10$, and $\mathrm{BH}_{4}$ systems play a major role in limiting oxidative damage during ferroptosis. However, the process and function of ferroptosis needs to be explored. An unresolved central issue is that these oxidative damage, antioxidant defense mechanisms, and membrane repair mechanisms are also involved in the regulation of other kinds of non-ferroptotic cell death (Bae et al., 2011; Liu et al., 2020a). Thus, although they may share a common upstream mechanism, the identification of unique downstream effectors may distinguish ferroptosis from non-ferroptotic cell death. Similarly, it remains a challenge to distinguish the pathological role of ferroptosis and non-ferroptotic cell death in disease (Hotchkiss et al., 2009). In addition, the complexity of autophagy and lipid metabolism in the regulation of ferroptosis should be further clarified (Liu et al., 2020b; Xie et al., 2020b) and therefore provide a reasonable explanation for regulating ferroptosis in a context-dependent manner.

\section{AUTHOR CONTRIBUTIONS}

All authors conceptualized and wrote the manuscript.

\section{ACKNOWLEDGMENTS}

We thank Dave Primm (Department of Surgery, University of Texas Southwestern Medical Center) for his critical reading of the manuscript.

Bae, Y. S., Oh, H., Rhee, S. G., and Yoo, Y. D. (2011). Regulation of reactive oxygen species generation in cell signaling. Mol. Cells 32, 491-509. doi: 10.1007/s10059011-0276-3

Bai, Y., Meng, L., Han, L., Jia, Y., Zhao, Y., Gao, H., et al. (2019). Lipid storage and lipophagy regulates ferroptosis. Biochem. Biophys. Res. Commun. 508, 997-1003. doi: 10.1016/j.bbrc.2018.12.039 
Basit, F., van Oppen, L. M., Schockel, L., Bossenbroek, H. M., van Emst-de Vries, S. E., Hermeling, J. C., et al. (2017). Mitochondrial complex I inhibition triggers a mitophagy-dependent ROS increase leading to necroptosis and ferroptosis in melanoma cells. Cell Death Dis. 8:e2716. doi: 10.1038/cddis.2017.133

Bedard, K., and Krause, K. H. (2007). The NOX family of ROS-generating NADPH oxidases: physiology and pathophysiology. Physiol. Rev. 87, 245-313. doi: 10. 1152/physrev.00044.2005

Bersuker, K., Hendricks, J. M., Li, Z., Magtanong, L., Ford, B., Tang, P. H., et al. (2019). The CoQ oxidoreductase FSP1 acts parallel to GPX4 to inhibit ferroptosis. Nature 575, 688-692. doi: 10.1038/s41586-019-1705-2

Canli, O., Alankus, Y. B., Grootjans, S., Vegi, N., Hultner, L., Hoppe, P. S., et al. (2016). Glutathione peroxidase 4 prevents necroptosis in mouse erythroid precursors. Blood 127, 139-148. doi: 10.1182/blood-2015-06-654194

Chen, C. C., Liou, S. W., Chen, C. C., Chen, W. C., Hu, F. R., Wang, I. J., et al. (2011). Coenzyme Q10 reduces ethanol-induced apoptosis in corneal fibroblasts. PLoS One 6:e19111. doi: 10.1371/journal.pone.0019111

Chen, D., Tavana, O., Chu, B., Erber, L., Chen, Y., Baer, R., et al. (2017). NRF2 is a major target of ARF in p53-independent tumor suppression. Mol. Cell 68, 224.e4-232.e4. doi: 10.1016/j.molcel.2017.09.009

Chen, M. S., Wang, S. F., Hsu, C. Y., Yin, P. H., Yeh, T. S., Lee, H. C., et al. (2017). CHAC1 degradation of glutathione enhances cystine-starvationinduced necroptosis and ferroptosis in human triple negative breast cancer cells via the GCN2-eIF2alpha-ATF4 pathway. Oncotarget 8, 114588-114602. doi: 10.18632/oncotarget.23055

Chen, X., Li, J., Kang, R., Klionsky, D. J., Tang, D. (2020). Ferroptosis: machinery and regulation. Autophagy 1-28. doi: 10.1080/15548627.2020.1810918 [Epub ahead of print].

Dai, C., Chen, X., Li, J., Comish, P., Kang, R., and Tang, D. (2020a). Transcription factors in ferroptotic cell death. Cancer Gene Ther. doi: 10.1038/s41417-0200170-2 [Epub ahead of print].

Dai, E., Han, L., Liu, J., Xie, Y., Kroemer, G., Klionsky, D. J., et al. (2020b). Autophagy-dependent ferroptosis drives tumor-associated macrophage polarization via release and uptake of oncogenic KRAS protein. Autophagy 16, 1-15. doi: 10.1080/15548627.2020.1714209

Dai, E., Meng, L., Kang, R., Wang, X., and Tang, D. (2020c). ESCRT-III-dependent membrane repair blocks ferroptosis. Biochem. Biophys. Res. Commun. 522, 415-421. doi: 10.1016/j.bbrc.2019.11.110

Dai, E., Zhang, W., Cong, D., Kang, R., Wang, J., and Tang, D. (2020d). AIFM2 blocks ferroptosis independent of ubiquinol metabolism. Biochem. Biophys. Res. Commun. 523, 966-971. doi: 10.1016/j.bbrc.2020.01.066

Chen, X., Xu, S., Zhao, C., and Liu, B. (2019). Role of TLR4/NADPH oxidase 4 pathway in promoting cell death through autophagy and ferroptosis during heart failure. Biochem. Biophys. Res. Commun. 516, 37-43. doi: 10.1016/j.bbrc. 2019.06.015

Chen, Y., Liu, Y., Lan, T., Qin, W., Zhu, Y., Qin, K., et al. (2018). Quantitative profiling of protein carbonylations in ferroptosis by an aniline-derived probe. J. Am. Chem. Soc. 140, 4712-4720. doi: 10.1021/jacs.8b01462

Chu, B., Kon, N., Chen, D., Li, T., Liu, T., Jiang, L., et al. (2019). ALOX12 is required for p53-mediated tumour suppression through a distinct ferroptosis pathway. Nat. Cell Biol. 21, 579-591. doi: 10.1038/s41556-019-0305-6

Conrad, M., Angeli, J. P., Vandenabeele, P., and Stockwell, B. R. (2016). Regulated necrosis: disease relevance and therapeutic opportunities. Nat. Rev. Drug Discov. 15, 348-366. doi: 10.1038/nrd.2015.6

Conrad, M., and Pratt, D. A. (2019). The chemical basis of ferroptosis. Nat. Chem. Biol. 15, 1137-1147. doi: 10.1038/s41589-019-0408-1

Daher, B., Parks, S. K., Durivault, J., Cormerais, Y., Baidarjad, H., Tambutte, E., et al. (2019). Genetic Ablation of the Cystine Transporter xCT in PDAC Cells Inhibits mTORC1, Growth, Survival, and Tumor Formation via Nutrient and Oxidative Stresses. Cancer Res. 79, 3877-3890. doi: 10.1158/0008-5472.CAN18-3855

Deng, G., Li, Y., Ma, S., Gao, Z., Zeng, T., Chen, L., et al. (2020). Caveolin-1 dictates ferroptosis in the execution of acute immune-mediated hepatic damage by attenuating nitrogen stress. Free Radic. Biol. Med. 148, 151-161. doi: 10.1016/ j.freeradbiomed.2019.12.026

Dixon, S. J., Lemberg, K. M., Lamprecht, M. R., Skouta, R., Zaitsev, E. M., Gleason, C. E., et al. (2012). Ferroptosis: an iron-dependent form of nonapoptotic cell death. Cell 149, 1060-1072. doi: 10.1016/j.cell.2012.03.042
Do Van B, Gouel, F., Jonneaux, A., Timmerman, K., Gele, P., Petrault, M., et al. (2016). Ferroptosis, a newly characterized form of cell death in Parkinson's disease that is regulated by PKC. Neurobiol. Dis. 94, 169-178. doi: 10.1016/j. nbd.2016.05.011

Doll, S., Freitas, F. P., Shah, R., Aldrovandi, M., da Silva, M. C., Ingold, I. A. G., et al. (2019). FSP1 is a glutathione-independent ferroptosis suppressor. Nature 575, 693-698. doi: 10.1038/s41586-019-1707-0

Doll, S., Proneth, B., Tyurina, Y. Y., Panzilius, E., Kobayashi, S., Ingold, I. M., et al. (2017). ACSL4 dictates ferroptosis sensitivity by shaping cellular lipid composition. Nat. Chem. Biol. 13, 91-98. doi: 10.1038/nchembio.2239

Dolma, S., Lessnick, S. L., Hahn, W. C., and Stockwell, B. R. (2003). Identification of genotype-selective antitumor agents using synthetic lethal chemical screening in engineered human tumor cells. Cancer Cell 3, 285-296. doi: 10.1016/s15356108(03)00050-3

Elmore, S. (2007). Apoptosis: a review of programmed cell death. Toxicol. Pathol. 35, 495-516. doi: 10.1080/01926230701320337

Fang, X., Wang, H., Han, D., Xie, E., Yang, X., Wei, J., et al. (2019). Ferroptosis as a target for protection against cardiomyopathy. Proc. Natl. Acad. Sci. U.S.A. 116, 2672-2680. doi: 10.1073/pnas.1821022116

Friedmann Angeli, J. P, Schneider, M., Proneth, B., Tyurina, Y. Y., Tyurin, V. A., Hammond, V. J., et al. (2014). Inactivation of the ferroptosis regulator Gpx4 triggers acute renal failure in mice. Nat. Cell Biol. 16, 1180-1191. doi: 10.1038/ ncb3064

Fulda, S., Gorman, A. M., Hori, O., and Samali, A. (2010). Cellular stress responses: cell survival and cell death. Int. J. Cell Biol. 2010:214074. doi: 10.1155/2010/ 214074

Galluzzi, L., Vitale, I., Aaronson, S. A., Abrams, J. M., Adam, D., Agostinis, P., et al. (2018). Molecular mechanisms of cell death: recommendations of the nomenclature committee on cell death 2018. Cell Death Differ. 25, 486-541. doi: 10.1038/s41418-017-0012-4

Gao, M., Yi, J., Zhu, J., Minikes, A. M., Monian, P., Thompson, C. B., et al. (2019). Role of mitochondria in ferroptosis. Mol. Cell 73, 354.e3-363.e3. doi: 10.1016/j.molcel.2018.10.042

Golstein, P., and Kroemer, G. (2007). Cell death by necrosis: towards a molecular definition. Trends Biochem. Sci. 32, 37-43. doi: 10.1016/j.tibs.2006.11.001

Hasegawa, M., Takahashi, H., Rajabi, H., Alam, M., Suzuki, Y., Yin, L., et al. (2016). ). Functional interactions of the cystine/glutamate antiporter, CD44v and MUC1-C oncoprotein in triple-negative breast cancer cells. Oncotarget 7, 11756-11769. doi: 10.18632/oncotarget.7598

Hayano, M., Yang, W. S., Corn, C. K., Pagano, N. C., and Stockwell, B. R. (2016). Loss of cysteinyl-tRNA synthetase (CARS) induces the transsulfuration pathway and inhibits ferroptosis induced by cystine deprivation. Cell Death Differ. 23, 270-278. doi: 10.1038/cdd.2015.93

Henderson, C. J., Otto, D. M., Carrie, D., Magnuson, M. A., McLaren, A. W., Rosewell, I., et al. (2003). Inactivation of the hepatic cytochrome P450 system by conditional deletion of hepatic cytochrome P450 reductase. J. Biol. Chem. 278, 13480-13486. doi: 10.1074/jbc.M212087200

Hernandez-Camacho, J. D., Bernier, M., Lopez-Lluch, G., and Navas, P. (2018). Coenzyme Q10 supplementation in aging and disease. Front. Physiol. 9:44. doi: 10.3389/fphys.2018.00044

Hirata, Y., Iwasaki, T., Makimura, Y., Okajima, S., Oh-Hashi, K., and Takemori, H. (2019). Inhibition of double-stranded RNA-dependent protein kinase prevents oxytosis and ferroptosis in mouse hippocampal HT22 cells. Toxicology 418, 1-10. doi: 10.1016/j.tox.2019.02.012

Hotchkiss, R. S., Strasser, A., McDunn, J. E., and Swanson, P. E. (2009). Cell death. N. Engl. J. Med. 361, 1570-1583. doi: 10.1056/NEJMra0901217

Hou, W., Xie, Y., Song, X., Sun, X., Lotze, M. T., Zeh, H. J. III, et al. (2016). Autophagy promotes ferroptosis by degradation of ferritin. Autophagy 12, 1425-1428. doi: 10.1080/15548627.2016.1187366

Ingold, I., Berndt, C., Schmitt, S., Doll, S., Poschmann, G., Buday, K., et al. (2018). Selenium utilization by GPX4 is required to prevent hydroperoxideinduced ferroptosis. Cell 172, 409.e21-422.e21. doi: 10.1016/j.cell.20 17.11 .048

Jelinek, A., Heyder, L., Daude, M., Plessner, M., Krippner, S., Grosse, R., et al. (2018). Mitochondrial rescue prevents glutathione peroxidase-dependent ferroptosis. Free Radic. Biol. Med. 117, 45-57. doi: 10.1016/j.freeradbiomed. 2018.01.019 
Jiang, L., Kon, N., Li, T., Wang, S. J., Su, T., Hibshoosh, H., et al. (2015). Ferroptosis as a p53-mediated activity during tumour suppression. Nature 520, 57-62. doi: 10.1038 /nature14344

Jin, S., Zhou, F., Katirai, F., and Li, P. L. (2011). Lipid raft redox signaling: molecular mechanisms in health and disease. Antioxid Redox Signal. 15, 1043-1083. doi: 10.1089/ars.2010.3619

Kagan, V. E., Mao, G., Qu, F., Angeli, J. P., Doll, S., Croix, C. S., et al. (2017). Oxidized arachidonic and adrenic PEs navigate cells to ferroptosis. Nat. Chem. Biol. 13, 81-90. doi: 10.1038/nchembio.2238

Kang, R., Zeng, L., Zhu, S., Xie, Y., Liu, J., Wen, Q., et al. (2018). Lipid peroxidation drives gasdermin d-mediated pyroptosis in lethal polymicrobial sepsis. Cell Host Microbe 24, 97.e4-108.e4. doi: 10.1016/j.chom.2018.05.009

Kraft, V. A. N., Bezjian, C. T., Pfeiffer, S., Ringelstetter, L., Muller, C., Zandkarimi, F., et al. (2020). GTP Cyclohydrolase 1/Tetrahydrobiopterin counteract ferroptosis through lipid remodeling. ACS Cent. Sci. 6, 41-53. doi: 10.1021/ acscentsci.9b01063

Lee, H., Zandkarimi, F., Zhang, Y., Meena, J. K., Kim, J., Zhuang, L., et al. (2020). Energy-stress-mediated AMPK activation inhibits ferroptosis. Nat. Cell Biol. 22, 225-234. doi: 10.1038/s41556-020-0461-8

Lewerenz, J., Hewett, S. J., Huang, Y., Lambros, M., Gout, P. W., Kalivas, P. W., et al. (2013). The cystine/glutamate antiporter system $\mathrm{x}(\mathrm{c})(-)$ in health and disease: from molecular mechanisms to novel therapeutic opportunities. Antioxid Redox Signal. 18, 522-555. doi: 10.1089/ars.2011.4391

Li, C., Zhang, Y., Liu, J., Kang, R., Klionsky, D. J., and Tang, D. (2020). Mitochondrial DNA stress triggers autophagy-dependent ferroptotic death. Autophagy 18, 1-13. doi: 10.1080/15548627.2020.1739447

Lin, P. L., Tang, H. H., Wu, S. Y., Shaw, N. S., and Su, C. L. (2020). Saponin formosanin C-induced ferritinophagy and ferroptosis in human hepatocellular carcinoma cells. Antioxidants 9:682. doi: 10.3390/antiox9080682

Linkermann, A., Skouta, R., Himmerkus, N., Mulay, S. R., Dewitz, C., De Zen, F., et al. (2014). ). Synchronized renal tubular cell death involves ferroptosis. Proc. Natl. Acad. Sci. U.S.A. 111, 16836-16841. doi: 10.1073/pnas.1415518111

Liu, J., Kang, R., and Tang, D. (2020a). ESCRT-III-mediated membrane repair in cell death and tumor resistance. Cancer Gene Ther. doi: 10.1038/s41417-0200200-0 [Epub ahead of print].

Liu, J., Kuang, F., Kroemer, G., Klionsky, D. J., Kang, R., and Tang, D. (2020b). Autophagy-dependent ferroptosis: machinery and regulation. Cell Chem. Biol. 27, 420-435. doi: 10.1016/j.chembiol.2020.02.005

Liu, Y., Wang, Y., Liu, J., Kang, R., and Tang, D. (2020c). Interplay between MTOR and GPX4 signaling modulates autophagy-dependent ferroptotic cancer cell death. Cancer Gene Ther. doi: 10.1038/s41417-020-0182-y [Epub ahead of print].

Liu, Y., Wang, Y., Liu, J., Kang, R., and Tang, D. (2020d). The circadian clock protects against ferroptosis-induced sterile inflammation. Biochem. Biophys. Res. Commun. 525, 620-625. doi: 10.1016/j.bbrc.2020.02.142

Llabani, E., Hicklin, R. W., Lee, H. Y., Motika, S. E., Crawford, L. A., Weerapana, E., et al. (2019). Diverse compounds from pleuromutilin lead to a thioredoxin inhibitor and inducer of ferroptosis. Nat. Chem. 11, 521-532. doi: 10.1038/ s41557-019-0261-6

Lovatt, M., Adnan, K., Kocaba, V., Dirisamer, M., Peh, G. S. L., and Mehta, J. S. (2020). Peroxiredoxin-1 regulates lipid peroxidation in corneal endothelial cells. Redox Biol. 30:101417. doi: 10.1016/j.redox.2019.101417

Lu, B., Chen, X. B., Hong, Y. C., Zhu, H., He, Q. J., Yang, B., et al. (2019). Identification of PRDX6 as a regulator of ferroptosis. Acta Pharmacol. Sin. 40, 1334-1342. doi: 10.1038/s41401-019-0233-9

Martin-Sanchez, D., Ruiz-Andres, O., Poveda, J., Carrasco, S., Cannata-Ortiz, P., Sanchez-Nino, M. D., et al. (2017). Ferroptosis, but not necroptosis, is important in nephrotoxic folic acid-induced AKI. J. Am. Soc. Nephrol. 28, 218-229. doi: 10.1681/ASN.2015121376

Matsushita, M., Freigang, S., Schneider, C., Conrad, M., Bornkamm, G. W., and Kopf, M. (2015). T cell lipid peroxidation induces ferroptosis and prevents immunity to infection. J. Exp. Med. 212, 555-568. doi: 10.1084/jem.20140857

Mayr, L., Grabherr, F., Schwarzler, J., Reitmeier, I., Sommer, F., Gehmacher, T., et al. (2020). Dietary lipids fuel GPX4-restricted enteritis resembling Crohn's disease. Nat. Commun. 11:1775. doi: 10.1038/s41467-020-1 5646-6
McBean, G. J. (2012). The transsulfuration pathway: a source of cysteine for glutathione in astrocytes. Amino Acids 42, 199-205. doi: 10.1007/s00726-0110864-8

Muller, T., Dewitz, C., Schmitz, J., Schroder, A. S., Brasen, J. H., Stockwell, B. R., et al. (2017). Necroptosis and ferroptosis are alternative cell death pathways that operate in acute kidney failure. Cell Mol. Life Sci. 74, 3631-3645. doi: 10.1007/s00018-017-2547-4

NaveenKumar, S. K., Hemshekhar, M., Kemparaju, K., and Girish, K. S. (2019). Hemin-induced platelet activation and ferroptosis is mediated through ROSdriven proteasomal activity and inflammasome activation: protection by melatonin. Biochim. Biophys. Acta Mol. Basis Dis. 1865, 2303-2316. doi: 10. 1016/j.bbadis.2019.05.009

Neitemeier, S., Jelinek, A., Laino, V., Hoffmann, L., Eisenbach, I., Eying, R., et al. (2017). BID links ferroptosis to mitochondrial cell death pathways. Redox Biol. 12, 558-570. doi: 10.1016/j.redox.2017.03.007

Ou, Y., Wang, S. J., Li, D., Chu, B., and Gu, W. (2016). Activation of SAT1 engages polyamine metabolism with p53-mediated ferroptotic responses. Proc. Natl. Acad. Sci. U.S.A. 113, E6806-E6812. doi: 10.1073/pnas.1607152113

Pagano, G., Aiello Talamanca, A., Castello, G., Cordero, M. D., d'Ischia, M., Gadaleta, M. N., et al. (2014). Current experience in testing mitochondrial nutrients in disorders featuring oxidative stress and mitochondrial dysfunction: rational design of chemoprevention trials. Int. J. Mol. Sci. 15, 20169-20208. doi: 10.3390/ijms151120169

Park, E. J., Park, Y. J., Lee, S. J., Lee, K., and Yoon, C. (2019). Whole cigarette smoke condensates induce ferroptosis in human bronchial epithelial cells. Toxicol. Lett. 303, 55-66. doi: 10.1016/j.toxlet.2018.12.007

Qi, W., Li, Z., Xia, L., Dai, J., Zhang, Q., Wu, C., et al. (2019). LncRNA GABPB1AS1 and GABPB1 regulate oxidative stress during erastin-induced ferroptosis in HepG2 hepatocellular carcinoma cells. Sci. Rep. 9:16185. doi: 10.1038/s41598019-52837-8

Ran, Q., Van Remmen, H., Gu, M., Qi, W., Roberts, L. J. II, Prolla, T., et al. (2003). Embryonic fibroblasts from Gpx4+/- mice: a novel model for studying the role of membrane peroxidation in biological processes. Free Radic. Biol. Med. 35, 1101-1109. doi: 10.1016/s0891-5849(03)00466-0

Riddick, D. S., Ding, X., Wolf, C. R., Porter, T. D., Pandey, A. V., Zhang, Q. Y., et al. (2013). NADPH-cytochrome P450 oxidoreductase: roles in physiology, pharmacology, and toxicology. Drug Metab. Dispos. 41, 12-23. doi: 10.1124/ dmd.112.048991

Schweichel, J. U., and Merker, H. J. (1973). The morphology of various types of cell death in prenatal tissues. Teratology 7, 253-266. doi: 10.1002/tera.1420070306

Shimada, K., Skouta, R., Kaplan, A., Yang, W. S., Hayano, M., Dixon, S. J., et al. (2016). Global survey of cell death mechanisms reveals metabolic regulation of ferroptosis. Nat. Chem. Biol. 12, 497-503. doi: 10.1038/nchembio.2079

Shintoku, R., Takigawa, Y., Yamada, K., Kubota, C., Yoshimoto, Y., Takeuchi, T., et al. (2017). Lipoxygenase-mediated generation of lipid peroxides enhances ferroptosis induced by erastin and RSL3. Cancer Sci. 108, 2187-2194. doi: $10.1111 /$ cas. 13380

Skouta, R., Dixon, S. J., Wang, J., Dunn, D. E., Orman, M., Shimada, K., et al. (2014). Ferrostatins inhibit oxidative lipid damage and cell death in diverse disease models. J. Am. Chem. Soc. 136, 4551-4556. doi: 10.1021/ja411006a

Song, X., Zhu, S., Chen, P., Hou, W., Wen, Q., Liu, J., et al. (2018). AMPK-mediated BECN1 phosphorylation promotes ferroptosis by directly blocking system Xc(-) Activity. Curr. Biol. 28, 2388.e5-2399.e5. doi: 10.1016/j.cub.2018.05.094

Stockwell, B. R., Friedmann Angeli, J. P, Bayir, H., Bush, A. I., Conrad, M., Dixon, S. J., et al. (2017). Ferroptosis: a regulated cell death nexus linking metabolism, redox biology, and disease. Cell 171, 273-285. doi: 10.1016/j.cell.2017. 09.021

Sun, X., Niu, X., Chen, R., He, W., Chen, D., Kang, R., et al. (2016a). Metallothionein-1G facilitates sorafenib resistance through inhibition of ferroptosis. Hepatology 64, 488-500. doi: 10.1002/hep.28574

Sun, X., Ou, Z., Chen, R., Niu, X., Chen, D., Kang, R., et al. (2016b). Activation of the p62-Keap1-NRF2 pathway protects against ferroptosis in hepatocellular carcinoma cells. Hepatology 63, 173-184. doi: 10.1002/hep. 28251

Tan, S., Schubert, D., and Maher, P. (2001). Oxytosis: a novel form of programmed cell death. Curr. Top. Med. Chem. 1, 497-506. doi: 10.2174/1568026013394741 
Tang, D., Kang, R., Berghe, T. V., Vandenabeele, P., and Kroemer, G. (2019). The molecular machinery of regulated cell death. Cell Res. 29, 347-364. doi: 10.1038/s41422-019-0164-5

Teran, E., Hernandez, I., Tana, L., Teran, S., Galaviz-Hernandez, C., Sosa-Macias, M., et al. (2018). Mitochondria and coenzyme Q10 in the pathogenesis of preeclampsia. Front. Physiol. 9:1561. doi: 10.3389/fphys.2018.01561

Tsurusaki, S., Tsuchiya, Y., Koumura, T., Nakasone, M., Sakamoto, T., Matsuoka, M., et al. (2019). Hepatic ferroptosis plays an important role as the trigger for initiating inflammation in nonalcoholic steatohepatitis. Cell Death Dis. 10:449. doi: 10.1038/s41419-019-1678-y

Wang, D., Peng, Y., Xie, Y., Zhou, B., Sun, X., Kang, R., et al. (2016). Antiferroptotic activity of non-oxidative dopamine. Biochem. Biophys. Res. Commun. 480, 602-607. doi: 10.1016/j.bbrc.2016.10.099

Wang, Y., Liua, Y., Liua, J., Kang, R., and Tang, D. (2020). NEDD4L-Mediated LTF protein degradation limits ferroptosis. Biochem. Biophys. Res. Commu. 13, 506-515. doi: 10.1021/acschembio.7b01082

Wang, Y., and Tang, M. (2019). PM2.5 induces ferroptosis in human endothelial cells through iron overload and redox imbalance. Environ. Pollut. 254:112937. doi: 10.1016/j.envpol.2019.07.105

Wei, M. C., Zong, W. X., Cheng, E. H., Lindsten, T., Panoutsakopoulou, V., Ross, A. J., et al. (2001). Proapoptotic BAX and BAK: a requisite gateway to mitochondrial dysfunction and death. Science 292, 727-730. doi: 10.1126/ science. 1059108

Wen, Q., Liu, J., Kang, R., Zhou, B., and Tang, D. (2019). The release and activity of HMGB1 in ferroptosis. Biochem. Biophys. Res. Commun. 510, 278-283. doi: 10.1016/j.bbrc.2019.01.090

Wenzel, S. E., Tyurina, Y. Y., Zhao, J., St Croix, C. M, Dar, H. H., Mao, G., et al. (2017). PEBP1 wardens ferroptosis by enabling lipoxygenase generation of lipid death signals. Cell 171, 628.e26-641.e26. doi: 10.1016/j.cell.2017.09.044

Werner, E. R., Blau, N., and Thony, B. (2011). Tetrahydrobiopterin: biochemistry and pathophysiology. Biochem. J. 438, 397-414. doi: 10.1042/BJ20110293

Wu, M., Xu, L. G., Li, X., Zhai, Z., and Shu, H. B. (2002). AMID, an apoptosis-inducing factor-homologous mitochondrion-associated protein, induces caspase-independent apoptosis. J. Biol. Chem. 277, 25617-25623. doi: 10.1074/jbc.M202285200

Wu, Y., Zhang, S., Gong, X., Tam, S., Xiao, D., Liu, S., et al. (2020). The epigenetic regulators and metabolic changes in ferroptosis-associated cancer progression. Mol. Cancer 19:39. doi: 10.1186/s12943-020-01157-x

Wu, Z., Geng, Y., Lu, X., Shi, Y., Wu, G., Zhang, M., et al. (2019). Chaperonemediated autophagy is involved in the execution of ferroptosis. Proc. Natl. Acad. Sci. U.S.A. 116, 2996-3005. doi: 10.1073/pnas.1819728116

Xie, Y., Hou, W., Song, X., Yu, Y., Huang, J., Sun, X., et al. (2016). Ferroptosis: process and function. Cell Death Differ. 23, 369-379. doi: 10.1038/cdd.2015.158

Xie, Y., Kang, R., Sun, X., Zhong, M., Huang, J., Klionsky, D. J., et al. (2015). Posttranslational modification of autophagy-related proteins in macroautophagy. Autophagy 11, 28-45. doi: 10.4161/15548627.2014.984267

Xie, Y., Kuang, F., Liu, J., Tang, D., and Kang, R. (2020a). DUSP1 blocks autophagydependent ferroptosis in pancreatic cancer. J. Pancreatol. 319, 322-332. doi: 10.1016/j.jconrel.2020.01.008

Xie, Y., Li, J., Kang, R., and Tang, D. (2020b). Interplay between lipid metabolism and autophagy. Front. Cell Dev. Biol. 8:431. doi: 10.3389/fcell.2020.00431

Xie, Y., Zhu, S., Song, X., Sun, X., Fan, Y., Liu, J., et al. (2017). The tumor suppressor p53 limits ferroptosis by blocking DPP4 activity. Cell Rep. 20, 1692-1704. doi: 10.1016/j.celrep.2017.07.055

Yagoda, N., von Rechenberg, M., Zaganjor, E., Bauer, A. J., Yang, W. S., Fridman, D. J., et al. (2007). RAS-RAF-MEK-dependent oxidative cell death involving voltage-dependent anion channels. Nature 447, 864-868. doi: 10. 1038/nature05859

Yang, J., Liu, X., Bhalla, K., Kim, C. N., Ibrado, A. M., Cai, J., et al. (1997). Prevention of apoptosis by $\mathrm{Bcl}-2$ : release of cytochrome $\mathrm{c}$ from mitochondria blocked. Science 275, 1129-1132. doi: 10.1126/science.275.5303.1129

Yang, M., Chen, P., Liu, J., Zhu, S., Kroemer, G., Klionsky, D. J., et al. (2019). Clockophagy is a novel selective autophagy process favoring ferroptosis. Sci. $A d v$. 5:eaaw2238. doi: 10.1126/sciadv.aaw2238
Yang, W. H., Ding, C. C., Sun, T., Rupprecht, G., Lin, C. C., Hsu, D., et al. (2019). The hippo pathway effector TAZ regulates ferroptosis in renal cell carcinoma. Cell Rep. 28, 2501.e4-2508.e4. doi: 10.1016/j.celrep.2019. 07.107

Yang, W. H., Huang, Z., Wu, J., Ding, C. C., Murphy, S. K., and Chi, J. T. (2020a). A TAZ-ANGPTL4-NOX2 axis regulates ferroptotic cell death and chemoresistance in epithelial ovarian cancer. Mol. Cancer Res. 18, 79-90. doi: 10.1158/1541-7786.MCR-19-0691

Yang, Y., Luo, M., Zhang, K., Zhang, J., Gao, T., Connell, D. O., et al. (2020b). Nedd4 ubiquitylates VDAC2/3 to suppress erastin-induced ferroptosis in melanoma. Nat. Commun. 11:433. doi: 10.1038/s41467-020-14324-X

Yang, W. S., Kim, K. J., Gaschler, M. M., Patel, M., Shchepinov, M. S., and Stockwell, B. R. (2016). Peroxidation of polyunsaturated fatty acids by lipoxygenases drives ferroptosis. Proc. Natl. Acad. Sci. U.S.A. 113, E4966-E4975. doi: 10.1073/pnas. 1603244113

Yang, W. S., SriRamaratnam, R., Welsch, M. E., Shimada, K., Skouta, R., Viswanathan, V. S., et al. (2014). Regulation of ferroptotic cancer cell death by GPX4. Cell 156, 317-331. doi: 10.1016/j.cell.2013.12.010

Yang, W. S., and Stockwell, B. R. (2008). Synthetic lethal screening identifies compounds activating iron-dependent, nonapoptotic cell death in oncogenicRAS-harboring cancer cells. Chem. Biol. 15, 234-245. doi: 10.1016/j.chembiol. 2008.02.010

Yoshida, M., Minagawa, S., Araya, J., Sakamoto, T., Hara, H., Tsubouchi, K., et al. (2019). Involvement of cigarette smoke-induced epithelial cell ferroptosis in COPD pathogenesis. Nat. Commun. 10:3145. doi: 10.1038/s41467-019-109917

Youle, R. J., and Strasser, A. (2008). The BCL-2 protein family: opposing activities that mediate cell death. Nat. Rev. Mol. Cell Biol. 9, 47-59. doi: 10.1038/nrm2308

Yuan, H., Li, X., Zhang, X., Kang, R., and Tang, D. (2016). Identification of ACSL4 as a biomarker and contributor of ferroptosis. Biochem. Biophys. Res. Commun. 478, 1338-1343. doi: 10.1016/j.bbrc.2016.08.124

Zeng, T., Deng, G., Zhong, W., Gao, Z., Ma, S., Mo, C., et al. (2020). Indoleamine 2, 3-dioxygenase lenhanceshepatocytes ferroptosis in acute immune hepatitis associated with excess nitrative stress. Free Radic. Biol. Med. 58, 974-986. doi: 10.1016/j.freeradbiomed.2020.01.009

Zhang, H., He, Y., Wang, J. X., Chen, M. H., Xu, J. J., Jiang, M. H., et al. (2020). miR30-5p-mediated ferroptosis of trophoblasts is implicated in the pathogenesis of preeclampsia. Redox Biol. 29: 101402. doi: 10.1016/j.redox.2019.101402

Zhang, Y. H., Wang, D. W., Xu, S. F., Zhang, S., Fan, Y. G., Yang, Y. Y., et al. (2018). Alpha-Lipoic acid improves abnormal behavior by mitigation of oxidative stress, inflammation, ferroptosis, and tauopathy in P301S Tau transgenic mice. Redox Biol. 14, 535-548. doi: 10.1016/j.redox.2017. 11.001

Zhang, Y., Shi, J., Liu, X., Feng, L., Gong, Z., Koppula, P., et al. (2018). BAP1 links metabolic regulation of ferroptosis to tumour suppression. Nat. Cell Biol. 20, 1181-1192. doi: 10.1038/s41556-018-0178-0

Zorov, D. B., Juhaszova, M., and Sollott, S. J. (2014). Mitochondrial reactive oxygen species (ROS) and ROS-induced ROS release. Physiol. Rev. 94, 909-950. doi: 10.1152/physrev.00026.2013

Zou, Y., Li, H., Graham, E. T., Deik, A. A., Eaton, J. K., Wang, W., et al. (2020) Cytochrome P450 oxidoreductase contributes to phospholipid peroxidation in ferroptosis. Nat. Chem. Biol. 16, 302-309. doi: 10.1038/s41589-0200472-6

Conflict of Interest: The authors declare that the research was conducted in the absence of any commercial or financial relationships that could be construed as a potential conflict of interest.

Copyright (c) 2020 Kuang, Liu, Tang and Kang. This is an open-access article distributed under the terms of the Creative Commons Attribution License (CC BY). The use, distribution or reproduction in other forums is permitted, provided the original author(s) and the copyright owner(s) are credited and that the original publication in this journal is cited, in accordance with accepted academic practice. No use, distribution or reproduction is permitted which does not comply with these terms. 